WIS-92/34/March-Ph

March, 1992

\title{
Symplectic Fusion Rings and their Metric
}

\author{
Doron Gepner And AdAm Schwimmer * \\ Department of Physics \\ Weizmann Institute of Science \\ Rehovot 76100, Israel
}

\begin{abstract}
The fusion of fields in a rational conformal field theory gives rise to a ring structure which has a very particular form. All such rings studied so far were shown to arise from some potentials. In this paper the fusion rings of the WZW models based on the symplectic group are studied. It is shown that they indeed arise from potentials which are described. These potentials give rise to new massive perturbations of superconformal hermitian symmetric models. The metric of the perturbation is computed and is shown to be given by solutions of the sinh-gordon equation. The kink structure of the theories is described, and it is argued that these field theories are integrable. The $S$ matrices for the fusion theories are argued to be non-minimal extensions of the $G_{k} \times G_{1} / G_{k+1} S$ matrices with the adjoint perturbation, in the case of $G=S U(N)$.
\end{abstract}

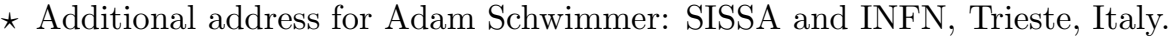


One of the central objects in the study of rational conformal field theory are the fusion rules which specify how the different fields in the theory fuse in the operator product algebra. For the WZW models the fusion rules were determined via a group theoretic criteria in ref. [1]. These assume the form

$$
C^{\lambda} C^{\mu}=\sum_{\nu} N_{\lambda, \mu}^{\nu} C^{\nu}
$$

where $N_{\lambda, \nu}^{\mu}$ are non-negative integers which express the number of times that the block of fields $C^{\nu}$ appear in the product. $\lambda, \nu, \mu$ denote highest weight vectors at level $k, \nu \theta \leq k$ where $k \geq 0$ is the integral coefficient of the Wess-Zumino term. Under this product, eq. (1), the primary fields form a commutative ring with a unit. In addition, the vacuum expectation value in the theory gives rise to an invariant bilinear form

$$
\left\langle C^{\lambda} C^{\mu}\right\rangle=\delta_{\lambda, \bar{\mu}}
$$

where $\bar{\mu}$ is the conjugate representation (charge conjugation). A connection between the fusion rules and the matrix of modular transformation was described in ref. [2].

A well known description for commutative rings is as a quotient of a free polynomial algebra modulo some relations, $P\left[x_{i}\right] /\left(p_{i}\right)$ where $p_{i}(x)$ denotes the relations among the generators $x_{i}$. Among such rings are the Jacobian varieties where the relations obey $p_{i}=\partial V(x) / \partial x_{i}$. The Jacobian rings are very special and the generic ring is far from being one ${ }^{\dagger}$. In ref. [3] the structure of the fusion rings of $S U(N)_{k}$ was described as a Schubert type calculus. It was shown that these rings are indeed Jacobian varieties with a potential which is

$$
V\left(x_{r}\right)=\sum_{i=1}^{N} q_{i}^{N+k}, \text { where } x_{r}=\sum_{i_{1}<i_{2}<\ldots<i_{r}} q_{i_{1}} q_{i_{2}} q_{i_{3}} \ldots q_{i_{r}}
$$

and $x_{N}=1$. For example, in the case of $S U(2), V$ becomes the Chebyshev polynomial of the first kind, $T_{k+2}(x)$ where $T_{n}(2 \cos \theta)=2 \cos (n \theta)$.

$\dagger$ We thank D. Kazhdan for a discussion of this point. 
The fact that the fusion ring is a Jacobian variety has a considerable geometrical significance. It implies essentially that it describes the moduli space of the complex manifold $V\left(x_{i}\right)=0$, where $x_{i}$ are any complex numbers, $x_{i} \in C$. Further, it allows one to describe it as a chiral algebra of a massive $N=2$ supersymmetric field theory, which are themselves geometrical in nature [3,4]. Basing on the known examples, it was conjectured in ref. [3] that all the fusion rings of rational conformal field theory are Jacobian varieties. If proven correct, this would allow one to classify all such conformal field theories via their potentials. In this note the fusion rings of the symplectic algebra $C_{n}$ are investigated. It is shown that they are indeed Jacobian varieties for any $n$ and any $k$, and the potential is described.

Let us recall theorem (4.1) in ref. [3]. According to it any fusion ring is of the form $P\left[x_{\alpha}\right] / I$ where $x_{\alpha}$ is a set of generating primary fields and the ideal $I$ can be described as the set of polynomials vanishing at the points

$$
x_{\alpha}=S_{\alpha, \beta}^{\dagger} / S_{0, \beta},
$$

where $\beta$ ranges over all the primary fields, and $S_{\alpha, \beta}$ is the matrix of modular transformations. Further, any ideal which vanishes precisely at these points is identical to $I$.

Thus, in order to establish that $I=\left(\partial_{i} V\right)$ where $V$ is some potential, it is enough to establish that the extrema of $V$ are precisely given by eq. (4). In the case of a current algebra theory based on the Lie algebra $G$, substituting the value of the matrix of modular transformations,

$$
S_{\lambda, \mu}=\left|\frac{M^{*}}{(k+g) M}\right|^{\frac{1}{2}} \sum_{w \in W}(-1)^{w} e^{-2 \pi i w(\lambda+\rho)(\mu+\rho) /(k+g)},
$$

we find that eq. (4) assumes the form

$$
x_{\lambda}=\frac{\sum_{w \in W}(-1)^{w} e^{2 \pi i w(\lambda+\rho)(\mu+\rho) /(k+g)}}{\sum_{w \in W}(-1)^{w} e^{2 \pi i w(\rho)(\mu+\rho) /(k+g)}},
$$

where $\lambda$ is one of the fundamental weights, $\mu$ is any weight at level $k, \rho$ is half the sum of positive roots, and $W$ denotes the Weyl group. Using the Weyl character 
formula, eq. (6) can be described as the specialization of the finite group character,

$$
x_{\lambda}=\operatorname{ch}_{\lambda}\left(\frac{\mu+\rho}{k+g}\right)
$$

where

$$
\operatorname{ch}_{\lambda}(\alpha)=\sum_{\mu \in L(\lambda)} e^{2 \pi i \mu \alpha}=\frac{\sum_{w \in W}(-1)^{w} e^{2 \pi i w(\lambda+\rho) \alpha}}{\sum_{w \in W}(-1)^{w} e^{2 \pi i w(\rho) \alpha}} .
$$

The problem of finding a fusion potential thus reduces to finding a polynomial whose extrema give the specialization points $(\mu+\rho) /(k+g)$. Note that this is equivalent to finding a fusion potential for the bosonic theory on the lattice $\sqrt{k+g} M_{l}$, where $M_{l}$ is the long root lattice, which is Weyl invariant [5]. Such a potential will give precisely these extrema points, where the weights which are not strictly dominant do not contribute since the Jacobian vanishes on them. Note however that it is crucial that the potential will be Weyl invariant, otherwise it cannot be expressed as a polynomial in the symmetric variables $x_{\alpha}$.

Let us consider then the bosonic models. Here we have $r$ free bosons propagating on a torus which is specified by the lattice $\sqrt{k} M$ where $M$ is an even lattice $\left(\alpha^{2}=2\right.$ for all $\alpha$ in $\left.M\right)$, which is generated by the vectors $l_{j}$. The primary fields are of the form $A_{p}=\exp (\vec{p} \vec{\phi} / \sqrt{k})$ and are specified by the set of the allowed momenta $\vec{p}$. Since this field must be single valued on the torus, it follows that $\vec{p} \in M^{*}$, where $M^{*}$ denotes the dual lattice, which is generated by $m_{i}$ where $m_{i} l_{j}=\delta_{i j}$. Now, since the dimension of the field $A_{p}$ is $p^{2} /(2 k)$ the extended algebra of the model consists of all the fields $A_{p}$ where $p \in M$. It follows that the primary fields are given by $p \in M^{*} \bmod k M$, or $A_{p}=A_{p+m}$ for any $p \in M^{*}$ and any $m \in k M$. The fusion rules for such fields are the addition of momenta (modulo $k M$ ),

$$
A_{p} A_{p^{\prime}}=A_{p+p^{\prime}} .
$$

Clearly the fusion ring is generated by $A_{m_{i}}$ where the $m_{i}$ are the generators of the dual lattice $M^{*}$. The fusion algebra is the group algebra over the abelian group 
$M^{*} \bmod k M$. Now, a well known theorem states that any abelian group is a direct sum of cyclical groups, and so,

$$
\frac{M^{*}}{k M} \approx Z_{n_{1}} \oplus Z_{n_{2}} \oplus \ldots \oplus Z_{n_{p}}
$$

where $Z_{n}$ stands for the order $n$ cyclical group and $n_{i} \mid n_{i+1}$. Now, let $\lambda_{i} \in M^{*}$ be the generator of the $Z_{n_{i}}$ group. Then since the fusion algebra is cyclical it follows that the relations in this algebra are,

$$
A_{\lambda_{i}}^{n_{i}}=1 \quad \text { for } i=1,2, \ldots, p
$$

Clearly, these relations can be integrated to a potential. Denoting by $x_{i}=A_{\lambda_{i}}$, we find that the relations eq. (11) are the derivatives with respect to $x_{i}$ of the potential,

$$
V=\sum_{i=1}^{p} \frac{x_{i}^{n_{i}+1}}{n_{i}+1}-x_{i}
$$

The extrema of $V$ are the solutions of the relations $x_{i}^{n_{i}}=1$. These can be parametrized by the elements $q \in M^{*} \bmod k M$,

$$
A_{p}=e^{2 \pi i p q / k}
$$

which is precisely the matrix of modular transformations for the bosonic model, in agreement with eq. (4). We conclude that any bosonic model is described by a potential which is given by eq. (12).

Now the affine model at level $k$ may be considered as a folding of the bosonic model, as is clear from eq. (6), where one takes the lattice to be the long root lattice of the group, $M_{l}$, at level $k+g$. Thus the problem of finding an affine fusion potential reduces to finding a Weyl invariant bosonic potential. Consider the symplectic algebra $C_{n}$. The simple roots of the algebra are given by $\alpha_{i}=$ $\frac{1}{\sqrt{2}}\left(\epsilon_{i}-\epsilon_{i+1}\right)$ for $i=1,2, \ldots, n-1$, and $\alpha_{n}=\sqrt{2} \epsilon_{n}$, where $\epsilon_{i}$ are orthogonal unit 
vectors. The long root lattice is spanned by $\sqrt{2} \epsilon_{i}$, whereas the dual of it is spanned by $\epsilon_{i} / \sqrt{2}$. It follows that $M^{*} \bmod k M_{l}$ is the abelian group $Z_{2 k}^{n}$. In agreement with eq. (13) the fusion variety is given by the points $q \in M^{*} \bmod k M_{l}$ according to $A_{p}=e^{2 \pi i p q}$. Denoting by $q=\sum r_{i} \epsilon_{i} /(\sqrt{2} k)$, where $r_{i}=0,1,2, \ldots, 2 k-1 \bmod 2 k$, we find that

$$
q_{i}=A_{\epsilon_{i} / \sqrt{2}}=e^{2 \pi i r_{i} /(2 k)}
$$

and there are $(2 k)^{n}$ solutions.

Let us look now for a potential which has precisely these points as extrema. Let $q_{i}=\exp \left(\epsilon_{i} / \sqrt{2}\right)$. Consider the potential

$$
V=\sum_{i} q_{i}^{n+k+1}+q_{i}^{-n-k-1},
$$

which is the character of the fundamental representation to the $(n+k+1)$ th 'power', in analogy with the $S U(N)$ case. Note that we shifted $k$ to $n+k+1$ in view of the application to the affine case. The extrema points of $V$ are given by $\partial V / \partial q_{i}=0$, or

$$
(k+n+1) q_{i}^{k+n}-(k+n+1) q_{i}^{-n-k-2}=0 .
$$

and coincide precisely with the points of $M^{*} \bmod (k+n+1) M_{l}$, eq. (14). Further, this potential has the essential property that it is invariant under the action of the Weyl group, which is of the form $\epsilon_{i} \rightarrow \pm \epsilon_{p(i)}$ where $p$ is any permutation. This is clear as $V$ is a scaled character of a representation.

Next, we need to express the potential $V$ in terms of Weyl invariant variables, which are the generators of the affine fusion ring. These we take, as mentioned earlier, to be the fundamental representations expressed in terms of their characters,

$$
x_{\Lambda}=\sum_{\lambda \in L(\Lambda)} e^{\lambda \vec{\theta}},
$$

where $\Lambda$ is any fundamental weight, and $L(\Lambda)$ is the set of weights in the represen- 
tation, and $\vec{\theta}$ is some specialization. An equivalent set of generators is,

$$
y_{r}=2^{r} \sum_{i_{1}<i_{2}<\ldots<i_{r}} \cos \theta_{i_{1}} \cos \theta_{i_{2}} \ldots \cos \theta_{i_{r}}
$$

for $r=1,2, \ldots, n$. The angular variables $\theta_{i}$ are defined by $2 \cos \theta_{i}=q_{i}+q_{i}^{-1}$. Note that $y_{r}$ is identical with the character $x_{r}=\operatorname{ch}_{\Lambda^{r}}\left(\epsilon_{i} \theta_{i} \sqrt{2}\right)$, eq. (17), where $\Lambda_{r}$ is fundamental weight, up to terms which are of lower order in $q$, i.e., $y_{r}=$ $x_{r}+f\left(x_{1}, x_{2}, \ldots, x_{r-1}\right)$ where $f$ is some function. It follows that $x_{r}$ and $y_{r}$ are an equivalent set of generators, with a Jacobian between them which is equal to one. The Jacobian for the change of variables $y_{r} \rightarrow \theta_{r}$ is easy to compute using the Vandermonde determinant,

$$
J=\frac{\partial y_{r}}{\partial \theta_{i}}=2^{n(n+1) / 2} \prod_{r=1}^{n} \sin \theta_{r} \prod_{1 \leq i<j \leq n}\left(\cos \theta_{i}-\cos \theta_{j}\right)
$$

Note that the Jacobian is anti-invariant with respect to the Weyl group, $\theta_{i} \rightarrow$ $\pm \theta_{p(i)}$. Thus it must be proportional to the denominator of the Weyl character formula, which is the unique generator of Weyl anti-invariant polynomials,

$$
D=\sum_{w \in W}(-1)^{w} e^{w(\rho) \theta_{i} \epsilon_{i} \sqrt{2}}
$$

Comparing the leading term shows that the two are in fact identical, $D=J$. It follows that the Jacobian $J$ vanishes at precisely the weights $\lambda=\theta_{i} \epsilon_{i} \sqrt{2}$ which are fixed by a Weyl reflection $\lambda=w(\lambda)$ for some reflection $w$. Thus, these points are no longer extrema of the potential when expressed in terms of the symmetric variables,

$$
x_{r}=\frac{\sum_{w \in W}(-1)^{w} e^{2 \pi i \omega\left(\Lambda_{r}+\rho\right) \theta_{i} \epsilon_{i} \sqrt{2}}}{D} .
$$

Further, points in the variety $M^{*} \bmod (k+g) M_{l}$ which are related by an element of the Weyl group clearly give the same value for $x_{r}$. Thus the extrema of the 
potential

$$
V=2 \sum_{i=1}^{n} \cos (k+g) \theta_{i}
$$

when expressed in terms of the variables $x_{r}$ are given by the the Weyl anti-invariant points in the variety $\theta_{i}=r_{i} /(2 k+2 g)$ where $r_{i}=0,1, \ldots, 2(k+g)-1$. As is well known (e.g., ref. [6]), these points are in correspondence with the integrable highest weights at level $k$, denoted by $\Lambda$,

$$
\sum_{i} r_{i} \epsilon_{i} / \sqrt{2}=\frac{\Lambda+\rho}{k+g} .
$$

To make the correspondence explicit, let us choose the representatives $1 \leq r_{1}<$ $r_{2}<\ldots<r_{n} \leq k+n$. It is clear that these are representatives modulo $W$ of the extrema points. The fundamental weights of $C_{n}$ are $\Lambda_{r}=\left(\epsilon_{1}+\epsilon_{2}+\ldots+\epsilon_{r}\right) / \sqrt{2}$, and $\rho=\left[n \epsilon_{1}+(n-1) \epsilon_{2}+\ldots+\epsilon_{n}\right] / \sqrt{2}$. It follows that

$$
\sum_{i} r_{i} \epsilon_{n+1-i} / \sqrt{2}=\rho+\Lambda=\sum_{i} s_{i} \Lambda_{i}
$$

where $s_{i}=r_{i}-r_{i-1}-1$ label the fundamental weights. In particular, $\sum s_{i}=$ $r_{n}-n \leq k$, implying that $\Lambda=s_{i} \Lambda_{i}$ is an integrable highest weight at level $k$. Thus we find that the points of the variety on which the potential $V$ has an extrema, are in correspondence with the integrable highest weight fields at level $k$. Denoting a point by $l_{\Lambda}$, where $\Lambda$ is an integrable highest weight, the value of the primary field $\Omega$ on such a point is (from eq. (7))

$$
x_{\Omega}=\operatorname{ch}_{\Omega}\left(\frac{\Lambda+\rho}{k+g}\right)
$$

It follows that these are precisely the correct values for the fusion variety, and so this ring is indeed the fusion ring of $C_{n}$. 
As a consequence of the fact that the Jacobian is the same as the denominator, it follows that the representations in the theory form a system of orthogonal polynomials when expressed in terms of the generators, where the measure is equal (up to a constant) to the denominator of the Weyl formula. This follows from

$$
\begin{array}{r}
C \delta_{\lambda, \mu}=\int_{0}^{2 \pi} \ldots \int_{0}^{2 \pi} \prod_{i} d \phi_{i} \sum_{w, w^{\prime}}(-1)^{w w^{\prime}} e^{2 \pi i w(\lambda+\rho) \phi_{i} \epsilon_{i} \sqrt{2}} e^{2 \pi i w^{\prime}(\mu+\rho) \phi \epsilon_{i} \sqrt{2}}= \\
\int_{0}^{2 \pi} \ldots \int_{0}^{2 \pi} \prod \mathrm{d} \phi_{i} \operatorname{ch}_{\lambda}\left(\phi_{i}\right) \operatorname{ch}_{\mu}\left(\phi_{i}\right) D^{2}\left(\phi_{i}\right),
\end{array}
$$

where $C$ is some constant. Changing variables to $x_{r}$ and remembering that the Jacobian is $D\left(\phi_{i}\right)$ it follows

$$
\int_{C} D\left(x_{i}\right) \prod_{i} \mathrm{~d} x_{i} \operatorname{ch}_{\lambda}\left(x_{i}\right) \operatorname{ch}_{\mu}\left(x_{i}\right)=C \delta_{\lambda, \mu} .
$$

where $\lambda$ and $\mu$ are any highest weights.

Let us turn now to examples. In the case of $C_{1} \approx S U(2)$ the potential we described for $C_{n}$, eq. (15), is immediately seen to be identical to that of $S U(2)$ described earlier, eq. (3), which is a Chebyshev polynomial of the first kind. The primary fields are given by Chebyshev polynomials of the second kind, $P_{m}(2 \cos \theta)=2 \sin (m+1) \theta / \sin \theta$ for $m=1,2, \ldots, k+1$. These indeed form an orthogonal polynomial system with a measure which is equal to the denominator, $D=\sqrt{4-x^{2}}$. Here $x$ stands for the fundamental representation.

Consider now the general case. In order to compute the potential $V\left(y_{i}\right)$ it is convenient to use the following recursion relation (in analogy with the $S U(N)$ case). The quantities $q_{i}=\exp \left(\theta_{i}\right)$ and $q_{i}^{-1}$ are the solutions of the polynomial equation,

$$
0=P=\prod_{i=1}^{n}\left(q-q_{i}\right)\left(q-q_{i}^{-1}\right)=\sum_{r=0}^{2 n}(-1)^{r} s_{r} q^{r} .
$$

The coefficients $s_{r}$ are Weyl-symmetric functions of the $q_{i}$ and can be readily 
expressed in terms of the $x_{i}$, eq. (21). For example, in the case of $n=2\left(C_{2}\right)$ we find

$$
P=q^{4}-x_{1} q^{3}+\left(x_{2}+1\right) q^{2}-x_{1} q+1=0 .
$$

Multiplying eq. (28) by $q^{r}$ for some $r$ and summing over the solutions, we find the recursion relation for the potentials,

$$
\sum_{i=0}^{2 n}(-1)^{i} s_{i} V_{i+r}=0 .
$$

For $n=2$ this recursion relation becomes $V_{r+4}-x_{1} V_{r+3}+\left(1+x_{2}\right) V_{r+2}-x_{1} V_{r+1}+$ $V_{r}=0$. Substituting the two initial values $V_{0}=4, V_{1}=x_{1}$, using also $V_{r}=V_{-r}$, we find recursively the potentials, $V_{2}=x_{1}^{2}-2 x_{2}-2, V_{3}=x_{1}^{3}-3 x_{1} x_{2}, V_{4}=$ $x_{1}^{4}-4 x_{1}^{2} x_{2}+2 x_{2}^{2}+4 x_{2}-2, V_{5}=x_{1}^{5}-5 x_{1}^{3} x_{2}+5 x_{1} x_{2}^{2}+5 x_{1} x_{2}-5 x_{1}$, etc.

According to the foregoing discussion the fusion ring of $C_{n}$ at level $k$ is given by $P\left[x_{i}\right] / I_{k}$, where the ideal $I_{k}$ is generated by the derivatives of the potential $I_{k}=\left(\partial_{i} V_{k+g}\left(x_{i}\right)\right)$. For $n=2(g=3)$ we find at $k=1$ the derivatives $\partial_{x_{2}} V_{4}=$ $-4 x_{1}^{2}+4 x_{2}+4=0$ and $\partial_{x_{1}} V_{4}=4 x_{1}^{3}-8 x_{1} x_{2}=0$. Equivalently these relations are $x_{1} x_{2}=x_{1}$ and $x_{1}^{2}=x_{2}+1$. These are precisely the relations of the fusion ring of $C_{2} \approx B_{2}$ at level one, which are the same as the Ising model. For a general $k$ the following relations can be established,

$$
\begin{aligned}
& \frac{1}{k+3} \frac{\partial V_{k+3}}{\partial x_{2}}=-\chi_{k+1,0}\left(x_{1}, x_{2}\right) \\
& \frac{1}{k+3} \frac{\partial V_{k+3}}{\partial x_{1}}=x_{1} \chi_{k+1,0}\left(x_{1}, x_{2}\right)-\chi_{k, 1}\left(x_{1}, x_{2}\right)
\end{aligned}
$$

where $\chi_{n, m}\left(x_{1}, x_{2}\right)$ is the character of the representation with highest weight $n \Lambda_{1}+$ $m \Lambda_{2}$. It follows that the ideal $\left(\partial_{i} V_{k+3}\right)$ is generated by the characters $\chi_{k+1,0}$ and $\chi_{k, 1}$. These are precisely the two generators of the relations of the fusion ring. Similarly, this type of relation can be seen for $C_{n}$ with $n \geq 3$. 
The fusion potentials for $S U(N)$ as well as $C_{n}$ described above, have an alternative description as superpotentials of massive $N=2$ supersymmetric Wess-Zumino theories (or Landau-Ginsburg) theories. These are described by the lagrangian,

$$
\mathcal{L}=\int \mathrm{d} \theta \mathrm{d} \bar{\theta} K\left(x_{i}, x_{i}^{*}\right)+\int \mathrm{d} \theta V\left(x_{i}\right)+\text { c.c. }
$$

where $x_{i}$ is interpreted as an $N=2$ scalar chiral superfield. The chiral fields in the theory, again form an associative algebra, the relations of which are described by the equation of motion $\frac{\partial V}{\partial x_{i}}=0$. Thus we find that the operator products of the chiral fields are described by the same ring $P\left[x_{i}\right] /\left(\partial_{i} V\right)$. If we take $V$ to be a fusion potential, the products of the chiral fields would be described by the fusion coefficients. One can deform the $N=2$ supersymmetric field theory by adding the supersymmmetry partner of some chiral field in the theory. This changes the superpotential $V$ by adding to it the same chiral field, expressed as a polynomial in the $x_{i}$. The $N=2 \mathrm{LG}$ theory is conformally invariant if the potential $V$ admits some grading, i.e., a conserved $U(1)$ charge with some particular assignment for the charges of the generating chiral fields $x_{i}$ which appear in the lagrangian. Thus the fusion rings may be considered as massive perturbations of $N=2$ superconformal field theories. For example, the potential for $S U(2)_{4}$ is given by the fourth Chebyshev polynomial $T_{4}(x)=x^{4}-4 x^{2}+2$. The $k=2$ minimal model has a superpotential which is $V=x^{4}$. Thus the $S U(2)_{4}$ theory is a massive perturbation of the $k=2$ minimal model. Similarly, the $S U(N)_{k}$ fusion rings may be thought of as massive deformations [3,4] of the $N=2$ superconformal theories based on the hermitian symmetric space construction (h.s.s.) $\frac{S U(n+1) \times S O(2 n)}{S U(n) \times U(1)}$ described in ref. [7].

It turns out that the fusion rings of $C_{n}$ describe massive perturbations of the same $S U(n)$ hermitian symmetric spaces, though with entirely different perturbations. (Except, of course, in the case of $C_{1} \approx S U(2)$.) To see this, assume that the variables $q_{i}$ in eq. (14) all have a degree which is equal to one. The nonhomogenous terms in the potential for $q_{i}$ are due to the $q_{i}^{-1}$ terms in the potential 
and in the variables $x_{i}$. Thus going to the conformal limit amounts to dropping these terms, leaving us with the potential

$$
V=\sum_{i=1}^{n} q_{i}^{n+k+1}
$$

expressed in terms of the variables

$$
x_{r}=\sum_{i_{1}<i_{2}<\ldots<i_{r}} q_{i_{1}} q_{i_{2}} \ldots q_{i_{r}} .
$$

This is exactly the potential of the h.s.s. theory $S U(n+1)_{k} / S U(n) \times U(1)[3]$. The degree of the $x_{r}$ variable is $r$. It is not hard to see that the degrees of the perturbations in the theory are given by $d_{v}-2 r$ where $r$ is a positive integer and $d_{v}$ is the degree of the potential. This is in contrast with the $S U(n+1)_{k}$ fusion rings where the degree of the perturbations is $d_{v}-(n+1) r$. Thus, these are two very different perturbations.

As an example consider $\left(C_{2}\right)_{2}$. The potential for this theory is $V=x_{1}^{5}-5 x_{1}^{3} x_{2}+$ $5 x_{1} x_{2}^{2}+5 x_{1} x_{2}-5 x_{1}$. The quasi-homogenous part of this potential $V_{0}=x_{1}^{5}-5 x_{1}^{3} x_{2}+$ $5 x_{1} x_{2}^{2}$ is precisely the superpotential of the h.s.s. model $S U(3) / S U(2) \times U(1)$ at level $k=2$ (which is equivalent to the $k=10$ minimal model with the $D$ modular invariant). The rest is the perturbation which is $5 x_{1} x_{2}-5 x_{1}$.

There are reasons to believe that fusion rings lead to integrable $N=2$ supersymmetric models. The examples which were investigated include the bosonic models ref. [8] and the $S U(N)_{k}$ case [9] (Chebyshev perturbation). Thus the question arises whether this is true for the general fusion field theory, and in particular for the symplectic fusion rings described here. In order to examine this question, we will compute the metric along the perturbation and show that it is given as a solution of $\hat{A}_{1}^{n}$ Toda equation.

Let $|i\rangle$ denote the Ramond vacua of the theory. The metric is described by the amplitude, $g_{\bar{j}, i}=\langle\bar{j} \mid i\rangle$. Here $\langle\bar{j}|$ denotes the vacuum obtained from the $j$ state 
by spectral flow. Following ref. [4] let us denote by $C_{i}$ the matrix representing the chiral field $C_{i},\left(C_{i}\right)_{j k}=N_{j k}^{i}$ where $N_{j k}^{i}$ are the structure constants of the chiral algebra. For a one parameter potential $W=W\left(\lambda, x_{i}\right)$, the infinitesimal perturbation is $W_{\lambda}=\partial W / \partial \lambda$. For such a perturbation, the metric obeys the following differential equation $[10,4]$,

$$
\bar{\partial}\left(g \partial g^{-1}\right)+\left[W_{\lambda}, g W_{\lambda}^{\dagger} g^{-1}\right]=0
$$

where the equation is written in a matrix notation. In addition, one imposes the reality condition which follows from the transposition of chiral fields,

$$
\eta^{-1} g\left(\eta^{-1} g\right)^{*}=1
$$

where $\eta$ is the transpose pairing,

$$
\eta_{i, j}=\operatorname{Res} \frac{C_{i} C_{j}}{\prod_{i} \partial_{i} W}
$$

Consider the superpotential,

$$
W=\lambda V\left(x_{i}\right)
$$

where $V\left(x_{i}\right)$ is the potential for some fusion ring of a rational conformal field theory. The known such fusion potentials, as described above correspond to bosonic models and the $S U(N)$ and $C_{n}$ WZW models. As we shall shortly establish, in all such fusion rings, the metric assumes a very particular form and eq. (35) becomes an affine Toda equation for some root system. As we shall argue, this in turn is an indication of the integrability of the associated $N=2$ supersymmetric model, along this line of perturbation.

Now, for the fusion potential $W=\lambda V(x)$ one can choose as a basis for the chiral fields the actual primary fields of the theory, i.e., the polynomials which represent the primary fields in the original rational conformal field theory. Moreover, one 
can use this basis for all the values of the parameter $\lambda$. In this basis, rather strikingly, the metric is diagonal, $g_{\lambda, \mu}=0$ unless $\lambda=\mu$. This can be argued, on the basis of the fact that the metric is essentially a topological quantity and the topological theory still preserves the charge conjugation properties of the original rational conformal field theory. This can also be seen by a direct calculation using eq. (35). The technical reason for it is that $W$ acts as an external automorphism on the primary fields.

Consider the fusion potential $V\left(\phi_{i}\right)$. It has extrema at the points $\partial_{i} V=0$, which have the set of solutions $x_{1}, x_{2}, \ldots, x_{m}$. The point basis for the chiral algebra is defined by

$$
l_{i}\left(x_{j}\right)=\delta_{i, j}
$$

According to eq. (4) the primary fields are given in this basis by

$$
\Phi_{i}=\sum_{j} S_{i, j}^{\dagger} / S_{0, i} l_{j}
$$

where $\Phi_{i}$ is the primary field and $S$ is the matrix of modular transformations. In the point basis, the potential $V$ acts diagonally and has the value $V(x) l_{i}=V\left(x_{i}\right) l_{i}$. In the primary field basis it gives

$$
V(x) \Phi_{i}=\sum_{j} S_{i, j}^{\dagger} / S_{0, j} V\left(x_{j}\right) l_{j}
$$

Consider, for example, the $S U(N)$ potential eq. (3). Substituting the values of the variety we find that $V\left(x_{\lambda}\right)=\exp \left(2 \pi i \lambda \Lambda_{1}\right)$ where $\lambda$ is the integrable weight which correspond to the point, and $\Lambda_{1}$ is the first fundamental weight, or $V\left(x_{\lambda}\right)$ is given by the central element which correspond to $\lambda$. Using the relation

$$
S_{\lambda, \sigma(\mu)}^{\dagger}=e^{2 \pi i \Lambda_{\sigma} \lambda} S_{\lambda, \mu}^{\dagger}
$$

where $\sigma$ is an external automorphism and $\Lambda_{\sigma}$ is the corresponding fundamental 
weight, we find that eq. (41) assumes the form,

$$
V \Phi_{\mu}=\Phi_{\sigma(\mu)}
$$

where $\sigma$ is the generator of external automorphisms. Similarly, for a gaussian model, $V=x^{n+1} /(n+1)-x$, we find an analogous expression where $\sigma$ is replaced by the fusion automorphism, $\sigma(m)=m+1 \bmod n$. This particular form of the potential is responsible for the simplicity of the equation for the metric. Indeed we find that eq. (35) takes the form

$$
\bar{\partial}\left(g_{\mu} \partial g_{\mu}^{-1}\right)+g_{\mu}^{-1} g_{\sigma(\mu)}-g_{\sigma^{-1}(\mu)}^{-1} g_{\mu}=0
$$

Thus the metric is diagonal, and its flow involves only elements related by an external automorphism. Since $\sigma$ is an element of order $n$, generating a $Z_{n}$ cyclical group, it follows that eq. (44) is precisely the $\hat{A}_{n}$ Toda theory, as is seen by the change of variables $g_{\sigma^{i}(\mu)}=e^{\phi_{i}}$, for $i=0,1, \ldots, n$,

$$
\partial \bar{\partial} \phi_{i}=e^{\phi_{i+1}-\phi_{i}}-e^{\phi_{i}-\phi_{i-1}}
$$

which is an equivalent form of the $\hat{A}_{n}$ Toda theory. This was already established in [4] in a slightly different form.

Consider now the $C_{n}$ fusion potential, eq. (15). The extrema points are given by eq. (16), $q_{i}=\exp \left[\pi i r_{i} /(k+n+1)\right]$, where $r_{i}=0,1, \ldots, 2(k+n)+1$ modulo $k+n+1$. Thus, it follows that the values of the potential at these points are given by,

$$
V\left(r_{1}, r_{2}, \ldots, r_{n}\right)=\sum_{i=1}^{n}(-1)^{r_{i}}
$$

Now, unlike the previous cases, $V$ does not correspond to a central element. However, it still corresponds to an automorphism of the fusion rules. Remembering that the choice of representatives which corresponds to the integrable highest weights 
is $1<r_{1}<r_{2}<\ldots<r_{n}$, the automorphism assumes the form $r_{i} \rightarrow k+n+1-r_{i}$. Using the diagonal ansatz $g_{\lambda, \mu}=g_{\lambda} \delta_{\lambda, \mu}$ (which can be justified by an explicit calculation), along with the assumption that the metric factorizes into a product of the metrics for the separate $S U(2)$ factors that make this potential. $\left(C_{n}\right.$ appears here as a symmetrized product of the $n S U(2)$ 's, as follows from eq. (33)). For a connection with Chern-Simons-Witten theory see the appendix. Thus the equation for the metric assumes the form

$$
\begin{array}{r}
\bar{\partial}\left(g_{r_{1}, r_{2}, \ldots, r_{n}} \partial g_{r_{1}, r_{2}, \ldots, r_{n}}^{-1}\right)+\sum_{i=1}^{n} g_{r_{1}, r_{2}, \ldots, k+n+1-r_{i}, \ldots, r_{n}} g_{r_{1}, r_{2}, \ldots, r_{n}}^{-1}- \\
g_{r_{1}, r_{2}, \ldots, r_{n}} g_{r_{1}, r_{2}, \ldots, k+n+1-r_{i}, \ldots, r_{n}}^{-1}=0 .
\end{array}
$$

Consistency of the ansatz implies that the solution can be written as

$$
g_{r_{1}, r_{2}, \ldots, r_{n}}=\frac{1}{(\lambda \bar{\lambda})^{n / 2}} \prod_{i=1}^{n} e^{L\left(r_{i}\right)}
$$

where $L\left(r_{i}\right)$ is a solution of the sinh-gordon equation,

$$
\partial \bar{\partial} L\left(r_{i}\right)=2 \sinh \left[2 L\left(r_{i}\right)\right] .
$$

The transpose pairing $\eta$ is computed from eq. (37) and is found to be

$$
\eta_{\sigma, \mu}=\frac{1}{\lambda^{n}} \delta_{\sigma, \bar{\mu}}
$$

where $\bar{\mu}$ is defined as sending $r_{i}$ to $k+n+1-r_{i}$, for all $i$. The reality condition eq. (36) implies $L\left(r_{i}\right)=-L\left(k+n+1-r_{i}\right)$. The solutions of the sinh-gordon equation are classified by their boundary condition at the origin,

$$
L=\frac{1}{2} t \log z+\frac{1}{2} s+O\left(\lambda^{2-|t|} \bar{\lambda}^{2-|t|}\right)
$$

where $t$ and $s$ are some real constants, and $z=2 \sqrt{\lambda \bar{\lambda}}$. The condition for the 
solution to have no poles on the real axis $(\lambda \bar{\lambda}>0)$ is

$$
e^{s / 2}=\frac{1}{2^{t / 2}} \frac{\Gamma\left(\frac{1}{2}-\frac{t}{4}\right)}{\Gamma\left(\frac{1}{2}+\frac{t}{4}\right)}
$$

Since the solution is expected to have no singularities on the real axis, it remains only to fix the the value of $t(r)$ for $r=1,2, \ldots, k+n$ to specify it uniquely. This we do by examining the behaviour for $\lambda \rightarrow 0$. With the change of variables $x_{r} \rightarrow \lambda^{r /(k+n+1)} x_{r}$ the potential eq. (15) becomes that of the superconformal theory $S U(n+1) / S U(n)$ as discussed earlier, up to a perturbation which vanishes with $\lambda$. Under such a change of variables the primary field $P_{r_{1}, r_{2}, \ldots, r_{n}}$ becomes the field $\left[a_{1}, a_{2}, \ldots, a_{k}\right]$ in the h.s.s. theory where

$$
\left[a_{i}\right]=[1,1, \ldots, 1,2,2, \ldots, 2, \ldots, n, n, \ldots, n]
$$

and where $i$ appears $r_{i}-r_{i-1}-1$ times. When expressed in terms of the $x_{i}$ this field becomes the polynomial (Giambelli's formula)

$$
\left[a_{1}, a_{2}, \ldots, a_{k}\right]=\operatorname{det}_{1 \leq i<j \leq k} x_{a_{i}+i-j}
$$

This can be seen by directly examining the recursion relation for the polynomials $P_{r_{1}, r_{2}, \ldots, r_{n}}$ which is

$$
y_{r} P_{r_{1}, r_{2}, \ldots, r_{n}}=\sum_{\substack{i_{1}<i_{2}<\ldots<i_{r} \\ \pm}} P_{r_{1}, r_{2}, \ldots, r_{i_{1}} \pm 1, \ldots, r_{i_{2}} \pm 1, \ldots, r_{n}}
$$

where the \pm are summed over independently. This is a special case of the general recursion relation,

$$
\operatorname{ch}_{\lambda} \operatorname{ch}_{\mu}=\sum_{\rho \in L(\lambda)} \operatorname{ch}_{\mu+\rho}
$$

which holds for any group and any weights $\lambda$ and $\mu$ provided that $\mu$ is sufficiently large (i.e., having $\mu+\rho$ as a dominant weight for all $\rho$ ). Eq. (56) can be proved by 
writing explicitly the formula for the characters, using the Weyl character formula. The limit $\lambda \rightarrow 0$ corresponds precisely to taking the homogenous part of the Pierilike formula eq. (55), which then becomes precisely the classical Pieri formula, implying, in turn, the Giambelli formula eq. (54). This shows that indeed, at this limit, the $C_{n}$ polynomials are given by the determinant eq. (54).

From the fact that the two point function of this field is finite at the limit $\lambda \rightarrow 0$ it follows that

$$
g_{r_{1}, r_{2}, \ldots, r_{n}}=(\lambda \bar{\lambda})^{c / 3-q /(k+n+1)}\left\langle\left[a_{1}, a_{2}, \ldots, a_{k}\right] \mid\left[a_{1}, a_{2}, \ldots, a_{k}\right]\right\rangle
$$

where $c$ is the central charge of the theory, $c / 3=\sum_{i} 1 / 2-i /(k+n+1)$, and $q /(k+n+1)$ is the $U(1)$ charge of the field, $q=\sum a_{i}$. Thus we find for the boundary condition of the solution $L(r)$,

$$
\frac{t(r)}{4}=\frac{1}{2}-\frac{r}{k+n+1}
$$

and so the normalization becomes (using eq. 51-52),

$$
\left\langle\left[a_{1}, a_{2}, \ldots, a_{k}\right] \mid\left[a_{1}, a_{2}, \ldots, a_{k}\right]\right\rangle=\prod_{i=1}^{n} \frac{\Gamma\left(r_{i} /(k+n+1)\right)}{\Gamma\left(1-r_{i} /(k+n+1)\right)}
$$

This result for the normalizations of the h.s.s. superconformal theory agrees in the case of $n=1$ with those of the minimal models, the only ones known so far. In addition, the normalizations are explicitly dual, i.e., they remain invariant with the exchange of $n$ and $k$, provided one replaces the $r_{i}$ 's with the ones corresponding to the transpose of the original Young tableau. This is precisely the duality of the h.s.s. theory $S U(n+1)_{k} / S U(n) \times U(1)$.

Let us consider now the opposite limit where $\lambda \bar{\lambda} \rightarrow \infty$. In this limit the 
sinh-gordon equation behaves asymptotically as

$$
L(r)=-\sqrt{2 / \pi}(\lambda \bar{\lambda})^{-1 / 4} \sin [\pi t(r) / 4] e^{-4 \sqrt{\lambda \bar{\lambda}}}
$$

Now, in the point basis $l_{i}$, eq. (39), the metric assumes the form

$$
G_{\lambda, \mu}=S_{0, \lambda}^{\dagger} S_{0, \mu} \sum_{\rho} S_{\lambda, \rho}^{\dagger} S_{\mu, \rho} g_{\rho, \rho}
$$

where $G_{\lambda, \mu}=\left\langle l_{\lambda} \mid l_{\mu}\right\rangle$ is the point basis metric and $S$ is the matrix of modular transformations. $S$ may be written as

$$
S_{r_{i} ; m_{i}}=\sum_{p \in S_{n}}(-1)^{p} \prod_{i=1}^{n} \sin \left(\frac{r_{i} m_{p(i)}}{n+k+1}\right)
$$

up to an overall constant determined by unitarity, where $p \in S_{n}$ is any permutation and $(-1)^{p}$ is \pm 1 if $p$ is even or odd. Substituting the asymptotic form of $L$ and using the matrix $S$ we find the asymptotics of the metric in the point basis,

$$
g_{r_{i} ; m_{i}} \propto \sum_{p}(-1)^{p} \sum_{\gamma_{i}= \pm 1,0} e^{-4 \sqrt{\lambda \bar{\lambda}} \sum \gamma_{i}^{2}} \delta_{m_{p(i)}-r_{i}-\gamma_{i}}
$$

The asymptotics of the metric has a universal form [4]

$$
g_{i, j}=\frac{\beta_{i, j}}{(\lambda \bar{\lambda})^{n / 2}} e^{-2 M_{i, j}}
$$

where $M_{i, j}=\left|W\left(x_{i}\right)-W\left(x_{j}\right)\right|$ is the mass of the soliton interpolating between the two classical vacua described by the point basis [9], and $\beta_{i, j}$ is the transition amplitude between the vacua. We thus infer that in the $C_{n}$ massive theory there are $n$ different soliton masses which are $M_{s}=2 s$, for $s=1,2, \ldots, n$. Eq. (63) further describes the allowed transitions which are $r_{i} \rightarrow r_{i} \pm 1,0$ up to a permutation of the $r_{i}$, where there are exactly $s$ transitions for the $s$ soliton. Note that even though 
naively many other transitions could be allowed, resulting in other solitons, the calculation of the metric shows that these are the only ones present ${ }^{\star}$.

The kink structure that arises in the $N=2$ fusion theories described above affords the following common description. The set of ground states, being identical to the fusion variety described earlier, corresponds to the integrable highest weights at level $k$. The kinks that interpolate between these ground states, correspond to the tensor product with one of the fundamental weights, i.e., the weights $\lambda$ and $\mu$ are connected by a kink if and only if the fusion coefficient $N_{\lambda, \Lambda_{i}}^{\mu}$ is nonzero for some fundamental weight $\Lambda_{i}$. The different $\Lambda_{i}$ correspond to different kink masses. In the case of $S U(2)$ the calculation of the metric [4] shows indeed such a kink structure. For $S U(N)$ it is presumed that the solution of the affine Toda equation, eq. (45), does so as well, though a precise analysis is hindered by the lack of treatment of the boundary conditions. Finally, for $C_{n}$ the kink structure we calculated is identical with the multiplication by the different fundamental representations of $C_{n}$. This can be seen by noting that the soliton transition amplitude, eq. (63), is identical to the Giambelli formula, eq. (55) where the representation label, $r$ is identified with the $s$ soliton. Further, each representation corresponds to a distinct soliton mass, which is $2 s$. Thus it is natural to assume that for all the groups the kink structure of the fusion theory assumes this form. Each kink is described by some fundamental representation, and the allowed transitions are given by the fusion rules with that representation.

Further, knowing the kink structure of the theory, along with the fact that it is integrable, allows one to write down a consistent set of factorizable $S$ matrices which correspond to it, by solving the constraints of the Yang-Baxter equation, crossing symmetry and unitarity for the kink scattering theory. Amazingly, precisely the same kink structure described above was considered in ref.[11] where it was suggested to describe the perturbed coset models $G_{k} \times G_{1} / G_{k+1}$ by the field

\footnotetext{
* Note that the fact that $\beta_{i j}$ vanishes implies only that $g_{i j}$ is smaller asymptotically, through the subleading terms. These, however, correspond to multi-soliton transitions, and can be ignored in reading the solitonic structure.
} 
$\Phi_{\text {ad, } 0}$, in the case of $G=S U(N)$. In addition the masses of the kinks are the same as described here, being given by $\left|W\left(x_{i}\right)-W\left(x_{j}\right)\right|$. Since the analysis of ref. [11] depends only on the kink structure it follows that the $S$ matrices described there apply also for the fusion conformal field theories. At first this may seem puzzling since these are perturbations of different conformal field theories (i.e., the supersymmetric h.s.s. models) with a different central charge. The explanation of this is that while the minimal $S$ matrices indeed describe the perturbed $G_{k} \times G_{1} / G_{k+1}$ models, the solution allows additional factors, the so called $Z$ factors, that when appropriately chosen give the full $S$ matrix of the fusion theories. In fact, in the case of $S U(N)_{1}$ the perturbation becomes gaussian, and can be used to demonstrate this. As was discussed in ref. [8] the $S$ matrices are those of affine $\hat{A}_{n}$ Toda theories with some imaginary coupling, which are a non-minimal version of the $A_{n} S$ matrices. The minimal $S$ matrices of [11] reduce for $k=1$ to these $A_{n} S$ matrices. Thus, it is necessary only to determine the precise $Z$ factors for the $S$ matrices of ref. [11] to obtain the full $S$ matrices. This argumentation applies equally well for all other groups, where it is expected that the kinks will similarly correspond to the fundamental representations fusing between the classical vacua, which are given by the integrable weights at level $k$. The $S$ matrices still should be given by a quantum group construction, similar to the case of $S U(N)$. This will be discussed elsewhere ${ }^{\dagger}$. Note the triple role played here by the fusion rules. First they are used to define the potential and chiral algebra of the theory. In addition, the metric turns out to be diagonal in the primary field basis, for all the couplings. Finally, the kink structure of the theory and its associated $S$ matrix turns out to be given by the very same fusion rules!

The fact that the metric assumes this particular simple form is a considerable evidence for the fact that this fusion field theory is integrable. Note, in particular, the close analogy between the $C_{n}$ case and the $C_{1}=S U(2)$ case. The integrability of the latter has been investigated in ref. [9] at the level of finding conserved

\footnotetext{
$\dagger$ Further support for this picture is found in ref. [12] where the case of $S U(2)$ is treated in detail. Our suggested $S$ matrices agree with those of [12] for $S U(2)$.
} 
currents. It is expected that, similarly, conserved currents could be found for the $C_{n}$ theories described here.

This work on the symplectic fusion rings gives further support to two conjectures regarding the structure of fusion rings [3] and its connection to integrable $N=2$ supersymmetric theory [4]. Indeed, we find that the fusion rings of $C_{n}$ are described by a potential for any level, in accordance with the conjecture of ref. [3]. Further, these potentials lead to Toda equations for the metric, suggesting the integrability of the associated $N=2$ supersymmetric field theory.

Note added: After the completion of this paper we became aware of two related works. In ref. [13] the fusion potentials of $C_{n}$ are also described. In ref. [14] the lattice $S$ matrices associated to fusion rings are investigated. Our results appear to be in agreement with both works.

Acknowledgements: It is a pleasure for us to acknowledge discussions with S. Elitzur, D. Kazhdan, A.B. Zamolodchikov and Al.B. Zamolodchikov. The work of A. Schwimmer was supported in part by BSF grant no. 89-00140. D. Gepner wishes to thank the Rockefeller University and especially M. Evans and N. Khuri for the hospitality during the final stages of this work.

\section{APPENDIX A.}

\section{The connection with CSW theory.}

The fusion rings have a natural setting in the framework of Chern-SimonsWitten (CSW) theories [15]. Quantising the CSW theory for a group $G$ at level $k$ on a torus, one obtains a finite dimensional Hilbert space. This space has a very convenient description in terms of an effective quantum mechanical problem [16], in which the conjugate variables are $a_{1}$ and $a_{2}$,

$$
\left[a_{1}^{i}, a_{2}^{j}\right]=\frac{2 \pi i}{k+g} \delta^{i j}
$$

The indices $i, j$ take the values $1,2, \ldots, r$ where $r$ is the rank of the group. The 
wave functions of the states are

$$
\Psi_{\mu}^{e f f}\left(\vec{a}_{1}\right)=\sum_{w \in W}(-1)^{w} \delta^{P}\left(\vec{a}_{1}-\frac{w(\mu+\rho)}{k+g}\right)
$$

where

$$
\delta^{P}\left(\vec{a}_{1}-\vec{\ell}\right) \equiv \sum_{\alpha \in M_{l}} \delta\left(\vec{a}_{1}-\vec{\ell}-\alpha\right)
$$

and where $\mu$ is a weight and $M_{l}$ is the long root lattice. The independent states are in one to one correspondence with the integrable highest weight representations of the affine Lie algebra at level $k$. The natural 'gauge invariant' operators in the reduced phase space (i.e., invariant under shifts in $M_{l}$ and under the action of the Weyl group) are the Weyl characters

$$
x_{\lambda} \equiv \operatorname{ch}_{\lambda}\left(a_{1}\right)=\frac{\sum_{w \in W}(-1)^{w} e^{2 \pi i \omega(\lambda+\rho) a_{1}}}{\sum_{w \in W}(-1)^{w} e^{2 \pi i \omega(\rho) a_{1}}},
$$

where $\lambda$ is a fundamental weight. Similar operators can be defined in terms of $a_{2}$. The $x_{\lambda}$ are related to the Wilson loops on the torus in the full CSW theory. Obviously, the set of $x_{\lambda}$, for $\lambda$ which is a fundamental weight, generate a commutative ring generated by the $x_{\lambda}$ operators, which are expressed in terms of the commuting $a_{1}$. However, since the latter act on a finite dimensional Hilbert space, there are relations among the generators of this ring which fulfill 'secular equations'. These equations are of the general form,

$$
P\left(x_{\lambda_{1}}, \ldots, x_{\lambda_{r}}\right) \Psi_{\mu}=0
$$

for all the $\mu$ which are compatible with eq. (A.2). Therefore, the ideal is defined by a set of polynomials of $x_{\lambda}$ which have as solutions precisely all the $x_{\lambda_{i}}^{\mu}$ defined 
by

$$
x_{\lambda_{i}}^{\mu}=\operatorname{ch}_{\lambda_{i}}\left(\frac{\mu+\rho}{k+g}\right)
$$

The $\mathrm{SU}(2)$ potential is,

$$
V\left(x_{1}\right)=\frac{1}{2} \operatorname{ch}_{1}\left((k+2) a_{1}\right)=2 \cos \left(2 \pi \frac{(k+2) a_{1}}{\sqrt{2}}\right),
$$

where $k$ is the level. The corresponding polynomial equation is

$$
\frac{\sin \left(2 \pi \frac{(k+2) a_{1}}{\sqrt{2}}\right)}{\sin \left(2 \pi \frac{a_{1}}{\sqrt{2}}\right)}=0 .
$$

The action of the potential eq. (A.7) on an arbitrary representation

$$
c h_{m}\left(a_{1}\right) \equiv \frac{\sin \left(2 \pi \frac{(m+1) a_{1}}{\sqrt{2}}\right)}{\sin \left(2 \pi \frac{a_{1}}{\sqrt{2}}\right)},
$$

where $m=0,1,2, \ldots, k$, is

$$
\begin{aligned}
& V\left(x_{1}\right) \operatorname{ch}_{m}\left(a_{1}\right)=\cos \left(2 \pi \frac{(k+2) a_{1}}{\sqrt{2}}\right) \operatorname{ch}_{m}\left(a_{1}\right)- \\
& \cos \left(2 \pi \frac{(m+1) a_{1}}{\sqrt{2}}\right) \operatorname{ch}_{k+1}\left(a_{1}\right)=-\operatorname{ch}_{k-m}\left(a_{1}\right),
\end{aligned}
$$

where we used eq. (A.8) in order to add the second term. For SU(N) the potential is given by eq. (3). The polynomial equations give rise to the conditions

$$
q_{1}^{N+k}=q_{2}^{N+k}=. .=q_{N}^{N+k} \text { and } q_{1}^{N(N+k)}=1
$$

Using eq. (A.11) and the definition of the character eq. (A.4), the action of $V$ on any character can be easily calculated,

$$
V\left(\vec{a}_{1}\right) \operatorname{ch}_{\vec{\mu}}\left(\vec{a}_{1}\right)=N \operatorname{ch}_{\vec{\mu}+(N+k) \vec{\lambda}_{1}}\left(\vec{a}_{1}\right)
$$

where $\vec{\lambda}_{1}$ is the fundamental weight corresponding to the basic representation. The automorphism eq. (A.12) is cyclic of order $N$ due to the second condition in (A.11). 
For the symplectic groups $C_{n}$, using a decomposition of $\vec{a}_{1}$ in an orthogonal basis $\epsilon_{1}$, one finds a close connection with $S U(2)^{n}$. The potential is given by

$$
V\left(\vec{a}_{1}\right)=\sum_{i=1}^{n} \cos \left(2 \pi \frac{(k+n+1) a_{1}^{i}}{\sqrt{2}}\right)
$$

and therefore the polynomial equations are

$$
\operatorname{ch}_{k+n}\left(a_{1}^{i}\right)=0 \text { for } i=1, \ldots n \text {. }
$$

However, due to the presence of the Jacobian between the $C_{n}$ and the $S U(2)^{n}$ characters, eq. (A.14) acts on a Hilbert space completely antisymmetrised in terms of the $a_{1}^{i}$. We label a general irreducible representation of $C_{n}$ by the integer components $r_{i}$ in the $\frac{\epsilon_{i}}{\sqrt{2}}$ basis of the highest weight $\lambda$. Then the character of the representation becomes

$$
\operatorname{ch}_{\vec{\lambda}}\left(\vec{a}_{1}\right)=\frac{\sum_{P}(-1)^{P} \prod_{j=1}^{n} \operatorname{ch}_{r_{j}+n-j}\left(a_{1}^{j_{l}}\right)}{\sum_{P}(-1)^{P} \prod_{i=1}^{n} \operatorname{ch}_{n-i}\left(a_{1}^{i_{m}}\right)},
$$

where $P$ denotes the permutations of the $a_{1}^{i}$. Applying repeatedly eq. (A.12), we find the action of $V$ on the representations

$$
V_{\vec{\lambda}}\left(\vec{a}_{1}\right)=-\sum_{i=1}^{n} \operatorname{ch}_{\vec{\lambda}^{(i)}}\left(\vec{a}_{1}\right)
$$

where the components of $\vec{\lambda}^{(i)}$ are $r_{1}, . ., k+2 i-1-n-r_{i}, . ., r_{n}$, where $r_{1}, r_{2}, \ldots, r_{n}$ are the components of $\vec{\lambda}$. In order to scale the variables $x_{s} \equiv \operatorname{ch}_{\vec{\lambda}_{s}}$, where $\vec{\lambda}_{s}$ is the fundamental weight corresponding to $r_{1}=r_{2}=\ldots=r_{s}=1$, all the others $=0$, as $\lambda^{\frac{r}{k+n+1}}$, we have to scale the individual $S U(2)$ variables $\operatorname{ch}_{1}\left(a_{1}^{j}\right)$ as $\lambda^{\frac{1}{(k+n+1)}}$. This follows from the explicit expression of the character eq. (A.15). Therefore, in the conformal limit we obtain a $S U(2)^{n}$ theory defined, however, in the CSW formalism, completely antisymmetrised in the $a_{1}^{i}$ Hilbert space. Once this restriction is imposed, one can use factorisation in $S U(2)$ to simplify the calculation of the $C_{n}$ metric, as is done in the main text. 


\section{REFERENCES}

1. D. Gepner and E. Witten, Nucl. Phys. B278 (1986) 493.

2. E. Verlinde, Nucl. Phys. B300 [FS22] 360 (1988).

3. D. Gepner, Comm. Math. Phys. 141 (1991) 381.

4. S. Cecotti and C. Vafa, Nucl. Phys. B367 (1991) 359.

5. M. Crescimanno, MIT preprint, MIT-CTP-2021, October 1991.

6. V. Kac, "Infinite dimensional Lie algebras", Cambridge, Cambridge University Press (1985).

7. Y. Kazama and H. Suzuki, Nucl. Phys. B321 (1989) 232.

8. P. Fendley W. Lerche, S.D. Mathur and N.P. Warner, Nucl. Phys. B348 (1991) 66; W. Lerche and N.P. Warner, Nucl. Phys. B358 (1991) 571.

9. D. Nemeschansky and N.P. Warner, Caltech preprint, USC-91-031, October (1991)

10. A. Strominger, Com. Math. Phys. 133 (1990) 163

11. H.J. de Vega and V.A. Fateev, Int. Journal of Mod. Phys. A6 (1991) 3221

12. P. Fendley and K. Intriligator, BUHEP91, HUTP-91/A043, preprint, November (91)

13. M. Bourdeau, E.J. Mlawer, H. Riggs and H.J. Schnitzer, Brandeis University preprint, BRX-TH-327, October (1991).

14. D. Nemeschansky and N.P. Warner, to appear.

15. E. Witten, Comm. Math. Phys. 121 (1989) 351.

16. S. Elitzur, G. Moore, A. Schwimmer and N. Seiberg, Nucl. Phys. B326 (1989) 108. 